Geometry \& Topology

Volume 9 (2005) 375-402

Published: 3 March 2005

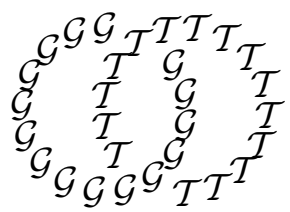

\title{
Kleinian groups and the rank problem
}

\author{
ILYA KAPOVICH \\ RICHARD WEIDMANN \\ Department of Mathematics, University of Illinois at Urbana-Champaign \\ 1409 West Green Street, Urbana, IL 61801, USA \\ and \\ Fachbereich Mathematik, Johann Wolfgang Goethe Universität \\ Robert Mayer-Straße 6-8, 60325 Frankfurt, Germany \\ Email: kapovich@math.uiuc.edu and rweidman@math.uni-frankfurt.de
}

\begin{abstract}
We prove that the rank problem is decidable in the class of torsion-free wordhyperbolic Kleinian groups. We also show that every group in this class has only finitely many Nielsen equivalence classes of generating sets of a given cardinality.
\end{abstract}

\section{AMS Classification numbers Primary: 20F67, 57M60}

Secondary: $30 \mathrm{~F} 40$

Keywords: Word-hyperbolic groups, Nielsen methods, 3-manifolds

Proposed: Walter Neumann

Seconded: Wolfgang Metzler, Cameron Gordon
Received: 31 August 2004

Accepted: 28 February 2005 


\section{Introduction}

If $G$ is a finitely generated group, the rank of $G$, $\operatorname{denoted} \operatorname{rk}(G)$, is the smallest cardinality of a subset $S \subseteq G$ such that $S$ generates $G$. The rank problem for a particular class of finitely presented groups asks if there is an algorithm that, given a finite presentation of a group $G$ from the class, computes the rank of $G$.

The rank problem is one of the more enigmatic and little understood grouptheoretic decision problems. For example, the word problem, the conjugacy problem and even (in the torsion-free case) the isomorphism problem [29] are solvable for the class of word-hyperbolic groups. On the other hand, by a theorem of Baumslag, Miller and Short [5], the rank problem is unsolvable for word-hyperbolic groups. The main ingredient in their proof is a remarkable construction due to Rips [26]. Namely that, given an arbitrary finitely presented group $Q$, there exists a short exact sequence

$$
1 \rightarrow K \rightarrow G \rightarrow Q \rightarrow 1
$$

such that $G$ is torsion-free, non-elementary, word-hyperbolic and such that $K$ is two-generated. Using the classical undecidability results, it is possible to create a family of finitely presented groups where it is undecidable whether $Q$ is trivial or not, and where every nontrivial $Q$ has rank at least three. Then the group $G$ based on $Q$ via the Rips construction can be generated by two elements if and only if $Q$ is trivial. Hence it is undecidable whether $\operatorname{rk}(G) \leq 2$ and therefore $\operatorname{rk}(G)$ is not computable.

A crucial feature of Rips' construction is that if both $K$ and $Q$ are infinite then $K$ is not quasiconvex in $G$. As it turns out, it is the presence of finitelygenerated non-quasiconvex subgroups that is often responsible for undecidability of various algorithmic problems in the context of word-hyperbolic groups. Thus Kapovich and Weidmann 22] proved that the rank problem is solvable for torsion-free locally quasiconvex word-hyperbolic groups.

However, some of the most important and interesting examples of word-hyperbolic groups come from the world of Kleinian groups and they are not necessarily locally quasiconvex. For instance, let $M$ be a closed hyperbolic 3-manifold fibering over a circle. In this case the fiber is a closed surface of negative Euler characteristic. Then $G=\pi_{1}(M)$ splits as a semi-direct product $G=H \ltimes \mathbb{Z}$, where $H$ is the fundamental group of a fiber. Thus $H$ is not quasiconvex in $G$.

The rank problem for 3-manifold groups is particularly interesting because of the connection between the rank and another important invariant, the Heegaard genus. Thus if $M$ is a closed 3-manifold, $G=\pi_{1}(M)$ and $h(M)$ is the 
Heegaard genus of $M$ then $\operatorname{rk}(G) \leq h(M)$. Waldhausen asked if in fact $\operatorname{rk}(G)$ is always equal to $h(M)$. Boileau and Zieschang [6] constructed a counterexample by producing a family of Seifert manifolds of Heegaard genus three with 2-generated fundamental groups. To this day the Waldhausen question remains open in the case of hyperbolic manifolds (see, for example, a paper of Dunfield and Thurston [9] where some experimental data is discussed).

The genus problem for 3-manifolds has been solved by Johannson [17] for sufficiently large 3-manifolds. Moreover he provides a procedure that produces all Heegaard splittings of a given genus. The case of small 3-manifolds has been claimed by Jaco and Rubinstein [16, 27 and relies on the existence and constructibility of so-called 1-efficient triangulations of irreducible atoroidal 3manifolds.

Definition 1.1 Let $\mathcal{M}$ be the class of all torsion-free word-hyperbolic groups $G$ such that $G$ admits a properly discontinuous isometric action on $\mathbb{H}^{3}$.

Our main result is:

Theorem A There exists an algorithm which, given a finite presentation of a group $G$ from the class $\mathcal{M}$, finds the rank of $G$.

The main technical tool needed for the proof of Theorem $\mathrm{A}$ is machinery developed by Kapovich and Weidmann in 21, 22] that provides a far-reaching generalization of Nielsen's methods in the general context of groups acting by isometries on Gromov-hyperbolic spaces. Another important ingredient is the "tameness conjecture" for Kleinian groups that has been recently proved by Agol [1] and, independently, by Calegari and Gabai [7. Together with the results of Canary [8] this yields a precise characterization of non-quasiconvex subgroups in the Kleinian groups context.

There are very few other classes of groups where the rank problem is known to be solvable. These include, in particular, the class of finitely generated nilpotent groups. For an arbitrary group $G$ it is easy to see that $\operatorname{rk}(G)=\operatorname{rk}(G / \mathcal{F}(G))$, where $\mathcal{F}(G)$ is the Frattini subgroup of $G$. If $G$ is finitely generated nilpotent, then the Frattini subgroup $\mathcal{F}(G)$ contains the commutator $[G, G]$. This implies that $\operatorname{rk}(G)=\operatorname{rk}\left(G_{\mathrm{ab}}\right)$ where $G_{\mathrm{ab}}=G /[G, G]$ is the abelianization of $G$. The rank problem is also decidable for torsion-free word-hyperbolic locally quasiconvex groups (Kapovich-Weidmann [22]), for (sufficiently large) Fuchsian groups [34] and for one-relator groups 24. The results of Arzhantseva and Ol'shanskii [4] show that, in a sense, the rank problem is "generically" solvable 
for finitely presented groups. The work of Weidmann 34] (see also KapovichWeidmann [22]) shows that the presence of torsion often creates a substantial difficulty for solving the rank problem. Indeed, there are many natural and seemingly easy to understand classes of finitely generated groups where the rank problem remains open. These include: virtually abelian groups, virtually free groups, virtually nilpotent groups, 3-manifold groups as well as lattices in $\mathbb{H}^{3}$.

We should stress that Theorem $\mathrm{A}$ is an abstract computability result. The nature of the proof is such that it cannot provide any complexity bound on the running time of the algorithm. In particular, the proof involves many "general enumeration" arguments, where several procedures are run in parallel, and at least one of them is guaranteed to eventually terminate, but where no complexity estimate is possible. We also stress that all the algorithms considered in this paper need to be uniform in all of their arguments, including the group presentation.

We also obtain:

Theorem B Let $G$ be a group from the class $\mathcal{M}$. Then for every $k \geq 1$ there are only finitely many Nielsen-equivalence classes of $k$-tuples generating $G$.

A similar but stronger statement was obtained by Kapovich and Weidmann 22, for torsion-free one-ended locally quasiconvex hyperbolic groups. Namely, for such a group $G$ for every $k \geq 1$ there are, up to conjugacy, only finitely many Nielsen-equivalence classes of $k$-tuples generating one-ended subgroups. This stronger statement is false in the presence of non-quasiconvex subgroups and in particular it fails for some groups from the class $\mathcal{M}$. Indeed, let $M$ be a closed hyperbolic 3-manifold fibering over a circle. Then $G=\pi_{1}(M)$ splits as a semi-direct product $G=H \ltimes\langle t\rangle$ where $H$ is the fundamental group of a fiber. If $k$ is the rank of $H$, then the subgroups $H_{n}:=\left\langle H, t^{n}\right\rangle$ are all $(k+1)-$ generated. However, each $H_{n}$ is normal of index $n$ in $G$ and hence $H_{n}$ is not conjugate to $H_{m}$ in $G$ for $m \neq n$. Thus there are, up to conjugacy, infinitely many Nielsen-equivalence classes of $(k+1)$-tuples generating subgroups of finite index in $G$.

More recently Souto 31] has obtained an interesting result that is relevant to the present article. Namely, he proved in 31] that if $\phi$ is a pseudo-Anosov homeomorphism of a closed oriented surface of genus $g \geq 2$, then the ranks of the fundamental groups of the mapping tori of sufficiently high powers of $\phi$ are equal to $2 g+1$ and that for these groups there is only one Nielsen equivalence 
class of generating $(2 g+1)$-tuples. His proof uses ideas closely related to those used in this article.

The proof of Proposition 5.3 about virtually cyclic groups is based on an argument explained to the first author by Derek Holt and we express special thanks to him. We are also grateful to Brian Bowditch, Misha Kapovich and Walter Neumann for helpful discussions about 3-manifold groups.

The first author was supported by the Max Planck Institute of Mathematics in Bonn and by NSF grant DMS-0404991.

\section{Algorithms in hyperbolic groups}

If $G$ is a group with a finite generating set $S$, we will denote by $X(G, S)$ the Cayley graph of $G$ with respect to $S$.

We refer the reader to [2, 10, 12, 14, 15, 20, for the basic background information regarding word-hyperbolic groups, quasiconvexity, Gromov hyperbolic spaces and their boundaries. Recall, however, that a subgroup $H$ of a word-hyperbolic group $G$ is quasiconvex in $G$ if for some (equivalently, for any) finite generating set $S$ of $G$ the subgroup $H$ is a quasiconvex subset of $X(G, S)$, that is, there is $\epsilon \geq 0$ such that for any $h_{1}, h_{2} \in H$ any geodesic $\left[h_{1}, h_{2}\right]$ in $X(G, S)$ is contained in the $\epsilon$-neighborhood of $H$.

In subsequent sections we will be relying on the following statement summarizing some known general algorithmic results regarding hyperbolic groups. Proposition 2.1 will be often used implicitly throughout the paper.

Proposition 2.1 The following statements hold:

(1) There is an algorithm that, given a finite presentation $G=\langle S \mid R\rangle$ of a word-hyperbolic group $G$, computes an integer $\delta \geq 0$ such that the Cayley graph $X=X(G, S)$ of $G$ with respect to $S$ is $\delta$-hyperbolic. Moreover:

(a) The algorithm then computes a finite state automaton accepting the language $L=L(G, S)$ of all short-lex geodesic words over $S$ for $G$. The algorithm then decides if $G$ is finite or infinite, and if $G$ is finite, the algorithm computes the order of $G$ and decides whether or not $G$ is virtually cyclic.

(b) Given an arbitrary word $w$ over $S$, the algorithm decides whether or not $w=1$ in $G$, and computes the order of the element of $G$ represented by $w$. 
(2) There is an algorithm with the following property.

(a) Given a finite presentation $G=\langle S \mid R\rangle$ of a word-hyperbolic group $G$ and a finite set $Q$ of words over $S$ generating a subgroup $H=\langle Q\rangle \leq G$, the algorithm computes an integer $C \geq 0$ such that $H$ is a $C$-quasiconvex subset of $X(G, S)$ if $H$ is quasiconvex in $G$, and runs forever if $H$ is not quasiconvex in $G$.

(b) If $H$ is quasiconvex in $G$, the algorithm also computes a finite state automaton $L_{H}=L_{H}(G, S)$ accepting all the short-lex geodesic words over $S$ representing elements of $H$.

(c) If $H$ is quasiconvex in $G$, the algorithm decides whether $H=G$, that is to say, whether $Q$ generates $G$.

(d) If $H$ is quasiconvex in $G$, the algorithm computes the index of $H$ in $G$.

(e) If $H$ is quasiconvex in $G$, the algorithm computes a finite presentation of $H$ on $S$.

(f) If $Q_{1}$ is another set of words over $S$ generating a quasiconvex subgroup $H_{1} \leq G$ (so that $H_{1} \cap H$ is also quasiconvex), the algorithm computes a finite generating set for $H_{1} \cap H$.

(g) If $H \leq G$ turns out to be quasiconvex, then, given an arbitrary word $w$ over $S$, the algorithm decides whether or not $w$ represents an element of $H$.

Proof The algorithm detecting hyperbolicity of a finite presentation $G=$ $\langle S \mid R\rangle$ and producing a hyperbolicity constant $\delta$ is due to Papasoglu [25. A general result of [10] states that, given a finite group presentation $G=\langle S \mid R\rangle$ known to possess a short-lex automatic structure with respect to $S$, one can algorithmically find such a structure. Since a word-hyperbolic group is short-lex automatic with respect to every finite generating set, this algorithm will always terminate if $G$ is word-hyperbolic.

The algorithm detecting quasiconvexity of a finitely generated subgroup is due to Kapovich [18. To decide if a hyperbolic group $G$ is virtually cyclic, we compute an automaton accepting the language $L(G, S)$ off all short-lex geodesic words over $S$ and check whether or not $L(G, S)$ has linear growth.

All of the other statements, except (2d), follow from basic well-known facts about word-hyperbolic groups.

To show that (2d) holds, suppose that $H$ is a quasiconvex subgroup of $G$. We may assume that $G$ is infinite since otherwise the problem is easily decidable. We need to compute the index of $H$ in $G$. Recall that all cyclic subgroups in a word-hyperbolic group are quasiconvex. 
For a quasiconvex subgroup $H$ of a word-hyperbolic group $G$ a result of Arzhantseva [3] implies that $[G: H]=\infty$ if and only if there is an element $g \in G$ of infinite order such that $\langle g\rangle \cap H=1$. Therefore we will run in parallel the following two procedures.

We will start the Todd-Coxeter coset enumeration process 30 for $H$ in $G$. If $[G: H]<\infty$, the process will eventually terminate and its output can be used to compute the index of $H$ in $G$.

In parallel, we will start enumerating all elements $g$ of $G$ (that is all words over $S)$. For each of them we check if $g$ has finite order or not. If $g$ has infinite order, compute the automaton accepting $L_{\langle g\rangle}$ and then an automaton accepting $L_{\langle g\rangle \cap H}=L_{\langle g\rangle} \cap L_{H}$. We then check whether or not $L_{\langle g\rangle \cap H}$ consists of only the empty word. If yes, we conclude that $\langle g\rangle \cap H=1$ and hence $[G: H]=\infty$. If not, we go on to the next $g$.

Eventually one of these procedures will terminate and we will have computed the index of $H$ in $G$.

We will further need the following simple lemma which applies in particular to the class of word-hyperbolic groups.

Lemma 2.2 Suppose $\mathcal{P}$ is a class of finitely presented groups with uniformly solvable word problem. There exists a partial algorithm with the following property.

Let $G$ be a group from $\mathcal{P}$ given by a finite presentation $G=\langle S \mid R\rangle$. Let $A \subset G$ be a finite subset of $G$ generating a subgroup $H$. Given a finite subset $Q \subseteq G$, the algorithm will eventually terminate if $H$ has finite index in $L:=\langle A, Q\rangle$ and it will run forever otherwise.

Suppose further that $H$ turns out to have finite index in $L$, that $H$ is given by a finite presentation on $A$ and that the pair $(H, G)$ comes from a class of subgroups of groups from $\mathcal{P}$ where the membership problem for $H$ in $G$ is solvable. Then the algorithm will also produce a finite presentation of $L$ on $A \cup Q$.

Proof Denote $B=A \cup Q$. Start enumerating all elements of $H$ (that is all words in $A$ ) and, in parallel, start enumerating all finite prefix-closed sets $W$ of words in $B$. For each such set $W=\left\{w_{1}, w_{2}, \ldots, w_{n}\right\}$, using this enumeration of $H$ and the algorithm solving the word problem in $G$, start checking if it is true that for every $w_{i}$ and every $b \in B^{ \pm 1}$ there is some $w_{j}$ such that $w_{i} b w_{j}^{-1}$ 
belongs to $H$, i.e. if $w_{i} b \in w_{j} H$. If yes, then, clearly, $H$ has finite index in $L$ as the set $W$ contains a representative of every coset of $H$ in $L$, i.e. as $L=\cup_{w \in W} H w$. Conversely, it is also obvious that if $H$ has finite index in $L$, the algorithm will eventually discover it and terminate.

Suppose now that $H$ was given by a finite presentation, that $G$ has solvable membership problem with respect to $H$ and that $L$ turns out to contain $H$ as a subgroup of finite index. Recall that the above algorithm produces a finite set $W$ such that $L=\cup_{w \in W} H w$. Using the algorithm solving the membership problem for $H$ in $G$, we can decide when $H w=H w^{\prime}$ and when $H w \neq H w^{\prime}$ for all $w, w^{\prime} \in W$. Using this information we can construct the Schreier coset graph for $H$ in $L$ with respect to the generating set $B$ of $L$. Combined with a finite presentation of $H$ this yields a finite presentation for $L$.

\section{Reduction to the one-ended case}

In this section we will show that it suffices to proof the main theorem for oneended groups.

Definition 3.1 Let $\mathcal{M}_{1}$ be the class of one-ended torsion-free groups $G$ such that $G$ admits a properly discontinuous convex-cocompact isometric action on $\mathbb{H}^{3}$.

It is clear that every group from $\mathcal{M}_{1}$ is word-hyperbolic so that $\mathcal{M}_{1} \subseteq \mathcal{M}$. Also, each of the classes $\mathcal{M}$ and $\mathcal{M}_{1}$ is closed under taking subgroups of finite index.

Moreover, it is a straightforward corollary of Thurston's Hyperbolization Theorem (see, for example, [23, Theorem 1.43]) that every one-ended group from $\mathcal{M}$ actually belongs to $\mathcal{M}_{1}$ :

Proposition 3.2 Let $G$ be a group acting isometrically and properly discontinuously on $\mathbb{H}^{3}$ and such that $G$ is torsion-free word-hyperbolic and one-ended. Then $G$ is isomorphic to the fundamental group of a compact hyperbolic 3manifold with (possibly empty) convex boundary. That is, $G$ belongs to $\mathcal{M}_{1}$.

The above fact reduces the rank problem for $\mathcal{M}$ to the rank problem for $\mathcal{M}_{1}$ because of Grushko's theorem, since by a result of Gerasimov [13] in the class of word-hyperbolic groups one can algorithmically compute a maximal free product decomposition of a group into freely indecomposable factors. 
Proposition 3.3 There exists an algorithm with the following properties. Given a finite presentation $G=\langle S \mid R\rangle$ of a torsion-free word-hyperbolic group $G$, the algorithm computes a number $r$ and finite presentations for groups $G_{1}, \ldots, G_{k}$ (with $k \geq 0$ ) such that

$$
G \cong G_{1} * \cdots * G_{k} * F_{r}
$$

and such that each $G_{i}$ is one-ended.

Proof A result of Gerasimov [13] shows that there is an algorithm that, given a finite presentation of a torsion-free word-hyperbolic group $G$, decides whether the group has 1,2 or infinitely many ends. If $G$ is two-ended, then $G$ is infinite cyclic. If $G$ is one-ended, then it is freely indecomposable.

If it turns out that $G$ has infinitely many ends we enumerate all finite presentations of $G$. This can be done by enumerating all possible finite group presentations $W$, all pairs of maps from the generators of $G$ to $W$ and from the generating set of $W$ to $G$, enumerating the normal closures of the relators of $G$ and $W$ and checking if the maps between $G$ and $W$ are group homomorphisms and if their compositions define identity maps for $G$ and $W$.

As $G$ is a proper free product we eventually find a presentation of type $\left\langle S_{1} \cup\right.$ $S_{2}\left|R_{1} \cup R_{2}\right\rangle$ such that any $r \in R_{i}$ is a word in $S_{i}^{ \pm 1}$ for $i=1,2$ and that $H=\left\langle S_{1}\right\rangle \neq 1$ and $K=\left\langle S_{2}\right\rangle \neq 1$. As both of these properties can be checked we eventually find the groups $H$ and $K$ with their finite presentations.

We then iterate the entire process (including determining the number of ends) by applying it to each of $H, K$ separately. Eventually we will get a collection of infinite cyclic and one-ended groups that gives a required decomposition of $G$.

\section{Virtual fibers and their recognition}

We need a precise description of non-quasiconvex finitely generated subgroups of groups from $\mathcal{M}_{1}$.

Definition 4.1 Two subgroups $H, K \leq G$ of a group $G$, are said to be commensurable in $G$ if $H \cap K$ has finite index in both $H$ and $K$.

Let $G$ be a group and $H \leq G$ be a subgroup. The commensurator $\operatorname{Comm}_{G}(H)$ of $H$ in $G$ is the set of all $g \in G$ such that $H$ and $g^{-1} H g$ are commensurable in $G$. 
The commensurator $\operatorname{Comm}_{G}(H)$ is easily seen to be a subgroup of $G$ containing $H$.

Definition 4.2 Let $G$ be a group from $\mathcal{M}_{1}$.

We will say that a subgroup $H \leq G$ is a fiber group for $G$ if $H$ is the fundamental group of a closed hyperbolic surface, $H$ is normal in $G$ and the quotient $G / H \cong \mathbb{Z}$ is infinite cyclic.

We will say that $H \leq G$ is a virtual fiber group for $G$ if $H$ is commensurable in $G$ with a subgroup $H_{1}$ where $H_{1}$ is a fiber group for some subgroup $G_{1} \leq G$ of finite index in $G$.

It is obvious that virtual fiber groups are not quasiconvex and, as it turns out, the converse is also true. The following statement is a corollary of the work of Canary [8] on geometrically infinite ends and of Marden's "tameness conjecture" for Kleinian groups recently proved by Agol [1] and Calegari-Gabai [7].

Theorem 4.3 Let $G$ be a group from $\mathcal{M}_{1}$ and let $H \leq G$ be a finitely generated subgroup. Then $H$ is quasiconvex in $G$ if and only if $H$ is not a virtual fiber group for $G$.

Remark 4.4 Note that virtual fibers are locally quasiconvex. Moreover, it follows from the results of Tukia 32 and Gabai [11] that if $P$ is virtually a closed hyperbolic surface group and $P$ is torsion-free then $P$ is a closed hyperbolic group itself. Thus virtual fibers are in fact surface groups. Hence the various algorithms discussed in Proposition 2.1. such as solving the uniform membership problem, computing quasiconvexity constants, etc, may be considerably improved and sped up in this case (see, for example 28]). Moreover, the "tameness conjecture" implies that the subgroup $H_{1}$ in the definition of a virtual fiber $H$ above can be taken as an actual topological fiber group of a finite cover $M_{1}$ fibering over a circle of a closed hyperbolic 3-manifold $M$ with the fundamental group $G$.

Convention 4.5 Computing a subgroup From now on to compute a wordhyperbolic subgroup $H$ of a word-hyperbolic group $G=\langle S \mid R\rangle$ shall mean to do all of the following:

(a) to find a finite generating set for $H$, a finite presentation for $H$ on that generating set, a hyperbolicity constant for that presentation and the order of $H$; 
(b) if $H$ is known to be quasiconvex in $G$, to find a quasiconvexity constant for $H$ in the Cayley graph of the ambient group $G$, the index of $H$ in $G$ and a finite state automaton accepting the language $L_{H}(G, S)$ of all short-lex representatives over $S$ of elements of $H$;

(c) if $H$ is known to have finite index in $G$, to find a right transveral for $H$ in $G$.

Proposition 4.6 There is an algorithm with the following properties.

Suppose we are given a finite presentation $G=\langle S \mid R\rangle$ of a group from $\mathcal{M}_{1}$, a finite subset $A \subseteq G$ and an element $g \in G$.

Then the algorithm decides whether the subgroup $H:=\langle A\rangle \leq G$ is quasiconvex in $G$ or not. If $H$ is quasiconvex, the algorithm also produces a quasiconvexity constant for $H$ in $X=X(G, S)$, a finite presentation for $H$ on $S$ and the index of $H$ in $G$.

If $H$ is not quasiconvex (and therefore is a virtual fiber group for $G$ ), the algorithm computes a subgroup $G_{1}$ of finite index in $G$ such that $H$ is commensurable with a fiber $H_{1}$ of $G_{1}$. The algorithm also computes a presentation of $G_{1}$ as a cyclic extension of $H_{1}$ and it computes a subgroup $H^{\prime} \leq H \cap H_{1}$ such that $H^{\prime}$ is of finite index in both $H$ and $H_{1}$.

The algorithm then decides whether or not $g \in H$.

Proof We will run the "detection of quasiconvexity" algorithm from Proposition 2.1 in parallel with the following procedure.

Start enumerating subgroups of finite index in $G$ and computing their presentations. For each such subgroup $G_{1} \leq G$ start checking if $G_{1}$ splits as a surface-by-cyclic group $G_{1}=H_{1} \ltimes \mathbb{Z}$, where $H_{1}$ is a surface group. This can be done by a "general enumeration" algorithm that lists all surface-by-cyclic presentations. For each such presentation $W$ start enumerating maps from the generating set of $W$ to $G_{1}$ and from the generating set of $G_{1}$ to $W$. For each pair of such maps, via enumeration of all the relations in $W$ and $G_{1}$, start checking if they define group homomorphisms and if their compositions define identity maps of $W$ and $G_{1}$ respectively. (We refer to this as the "general enumeration presentation comparison" argument.)

If so, then indeed $G_{1}=H_{1} \ltimes \mathbb{Z}$. Note that there is an algorithm which, for each $H_{1}$ above, solves the membership problem for $H_{1}$ in $G$. Now for each such $H_{1}$, using the algorithm from Lemma 2.2 start enumerating subgroups $H_{2}$ of $G$ 
containing $H_{1}$ as a subgroup of finite index and computing finite presentations for such subgroups $\mathrm{H}_{2}$.

For each of $H_{2}$ then start checking if $A \subseteq H_{2}$ (that is $H \leq H_{2}$ ). If yes, check if $H=\langle A\rangle \leq H_{2}$ has finite index in the surface group $H_{2}$. If yes, then $H$ is a virtual fiber group for $G$.

Since $H$ is either quasiconvex or is a virtual fiber group in $G$, eventually we will either detect the quasiconvexity of $G$ or will discover the fact that $H$ is a virtual fiber for $G$.

In either case it is easy to decide whether or not $g \in H$.

Thus we see, in particular, that there is a uniform algorithm for solving the uniform subgroup membership problem for groups from class $\mathcal{M}_{1}$.

\section{The relative rank with respect to virtual fibers}

Definition 5.1 Relative rank Let $G$ be a finitely generated group and let $Z \subseteq G$ be a subset. Define the relative rank $\operatorname{rk}_{Z}(G)$ of $G$ with respect to $Z$ as the smallest cardinality of a subset $S \subseteq G$ such that $G=\langle S \cup Z\rangle$.

The following statement is an elementary exercise.

Lemma 5.2 Let $D=\left\langle a, b \mid a^{2}=b^{2}=1\right\rangle$ be an infinite dihedral group.

(1) A $k$-tuple $\left(d_{1}, \ldots, d_{k}\right)$ generates $D$ if and only if $k \geq 2$ and this $k$-tuple is Nielsen-equivalent to $(a, b, 1, \ldots, 1)$.

(2) Suppose $s$ has order two in $D$ and $d_{1}, \ldots, d_{k} \in\langle a b\rangle$. Then $\left(s, d_{1}, \ldots, d_{k}\right)$ generates $D$ if and only $\left(d_{1}, \ldots, d_{k}\right)$ generates $\langle a b\rangle$, that is, if and only if, $\left(d_{1}, \ldots, d_{k}\right)$ is Nielsen-equivalent to $(a b, 1, \ldots, 1)$ in $D$.

Proposition 5.3 There is an algorithm that, given a finite presentation of a virtually cyclic group $G$ and a finite subset $Z \subseteq G$, finds $\operatorname{rk}_{Z}(G)$.

Proof We first compute the order of $G$ and the order of $L:=\langle Z\rangle$. If $G$ is finite or $L$ is infinite (that is $[G: L]<\infty$ ), the problem easily reduces to the relative rank problem for finite groups.

Therefore we may assume that $G$ is infinite and $L$ is finite. Then, by a wellknown fact about virtually cyclic groups, $G$ possesses a finite normal subgroup 
$N$ such that $\bar{G}:=G / N$ is either infinite cyclic or infinite dihedral. By a general enumeration argument we can find such a subgroup $N$ and determine which of these two possibilities occurs.

Case 1 The group $\bar{G}$ is infinite cyclic.

In this case clearly $N$ is the set of all torsion elements of $G$ and hence $L \leq N$. Find an element $x \in G$ such that $\bar{G}=\langle\bar{x}\rangle$.

By performing the Euclidean algorithm modulo $N$ we see that if a tuple $\left(g_{1}, \ldots, g_{k}, Z\right)$ generates $G$ then the $k$-tuple $\left(g_{1}, \ldots, g_{k}\right)$ is Nielsen-equivalent to a $k$-tuple of the form $\left(x n_{1}, n_{2}, \ldots, n_{k}\right)$ where $n_{i} \in N$.

To decide if $\operatorname{rk}_{Z}(G) \leq k$ we enumerate all tuples $\left(x n_{1}, n_{2}, \ldots, n_{k}, Z\right)$, where $n_{i} \in N$, and check if at least one of them generates $G$.

Case 2 The group $\bar{G}$ is infinite dihedral.

Then $\bar{G}=\left\langle\bar{s}, \bar{t} \mid \bar{s}^{2}=\bar{t}^{2}=1\right\rangle$. We compute $s, t \in G$ that map to $\bar{s}, \bar{t}$ accordingly. The infinite cyclic subgroup $\langle\overline{s t}\rangle$ has index two in $\bar{G}$. Let $H \leq G$ be the full preimage of $\langle\overline{s t}\rangle$ in $G$, so that $H=\langle s t, N\rangle$ has index two in $G$.

Note that $\bar{L}$ is either trivial or cyclic of order two. Suppose first that $\bar{L}$ is trivial, that is $Z \subseteq N$. Then by Lemma 5.2 if $\left(g_{1}, \ldots, g_{k}, Z\right)$ generates $G$ then $k \geq 2$ and $\left(g_{1}, \ldots, g_{k}\right)$ is Nielsen-equivalent to a $k$-tuple of the form $\left(s n_{1}, t n_{2}, n_{3}, \ldots, n_{k}\right)$ where $n_{i} \in N$. To decide if $\operatorname{rk}_{Z}(G) \leq k$ we enumerate all tuples $\left(s n_{1}, t n_{2}, n_{3} \ldots, n_{k}, Z\right)$, where $n_{i} \in N$, and check if at least one of them generates $G$.

Suppose now that $\bar{L}$ is cyclic of order two, that is there is some element $z \in Z$ such that $\bar{z}$ has order two. In this case $L \cap H \leq N$. Also, if $z^{\prime} \in Z$ is different from $z$, then either $z^{\prime} \in H$ (and hence $z^{\prime} \in N$ ) or $\overline{z^{\prime}}=\bar{z}$.

Suppose $\left(g_{1}, \ldots, g_{k}, Z\right)$ generates $G$. After multiplying $g_{i}$ by $z^{-1}$ if necessary, we obtain a tuple $\left(g_{1}^{\prime}, \ldots, g_{k}^{\prime}, Z\right)$ generating $G$ such that all $g_{i}^{\prime} \in H$. Note that $\left(\overline{g_{1}^{\prime}}, \ldots, \overline{g_{k}^{\prime}}, \bar{z}\right)$ generates $\bar{G}$ since for each $z^{\prime} \in Z$ either $\overline{z^{\prime}}=\bar{z}$ or $\overline{z^{\prime}}=1$. Now Lemma 5.2 implies that $\left(g_{1}^{\prime}, \ldots, g_{k}^{\prime}\right)$ is Nielsen-equivalent to $\left(\operatorname{stn}_{1}, n_{2}, \ldots, n_{k}\right)$ where $n_{i} \in N$.

To decide if $\operatorname{rk}_{Z}(G) \leq k$ we enumerate all tuples $\left(s t n_{1}, n_{2}, \ldots, n_{k}, Z\right)$ where $n_{i} \in N$, and check if at least one of them generates $G$.

Remark 5.4 The proof of Proposition 5.3 actually shows that if $G$ is virtually cyclic and $Z$ is a tuple generating a finite subgroup of $G$ then for every $k \geq 1$ there is a finite set $E$ of tuples $\left(f_{1}, \ldots, f_{k}, Z\right)$ such that every tuple $\left(g_{1}, \ldots, g_{k}, Z\right)$ generating $G$ is Nielsen-equivalent to a tuple from $E$. 
Lemma 5.5 Let $G \in \mathcal{M}_{1}$, let $G_{1}$ be a subgroup of finite index in $G$ and let $H \leq G_{1}$ be a fiber subgroup for $G_{1}$. Thus $G_{1}=H \ltimes\langle t\rangle$. Let $P \leq H$ be a subgroup of finite index in $H$ that is normal in $G_{1}$.

Then the following hold:

(1) For each subgroup $K$ of $G$ such that $P \leq K$, either $K$ has finite index in $G$ or $K$ contains $P$ as a subgroup of finite index.

(2) The set of subgroups conjugate to $P$ in $G$ is finite.

(3) For each $g \in G$ either $P$ has finite index in $L:=\left\langle P, g^{-1} P g\right\rangle$ (in which case $\left.g \in \operatorname{Comm}_{G}(P)\right)$ or $L$ has finite index in $G$.

(4) Either there is $g \in G-G_{1}$ such that $\left\langle P, g^{-1} P g\right\rangle$ has finite index in $G$ or for every $g \in G$ the subgroup $P$ has finite index in $\left\langle P, g^{-1} P g\right\rangle$ (in which case $\left.G=\operatorname{Comm}_{G}(P)\right)$.

Proof Let $g_{1}=1, \ldots, g_{n}$ be a right transversal for $G_{1}$ in $G$. Then every element of $G$ is uniquely expressible in the form $g=h t^{j} g_{i}$ where $1 \leq i \leq$ $n, j \in \mathbb{Z}$ and $h \in H$. Recall that $P$ is normal in $G_{1}$. Hence $g^{-1} P g=$ $g_{i}^{-1} t^{-j} h^{-1} P h t^{j} g_{i}=g_{i}^{-1} P g_{i}$. This shows that the set of conjugates of $P$ in $G$ is finite and part (2) of the lemma is established.

Let $K \leq G$ be a subgroup such that $P \leq K$ and suppose that $P$ has infinite index in $K$. Let $K_{1}=K \cap G_{1}$. Then $P \leq K_{1} \leq K$ and $K_{1}$ has finite index in $K$. Therefore $P$ has infinite index in $K_{1}$. Since $K_{1} \leq G_{1}$, every element of $K_{1}$ can be written in the form $t^{j} h$ where $j \in \mathbb{Z}$ and $h \in H$. Assume first that there is some element $a \in K_{1}$ of the form $a=t^{j} h$, where $j \neq 0$. Then it is easy to see that $\langle P, a\rangle$ has finite index in $G_{1}$ and hence in $G$, as required.

Suppose now that there is no element $a \in K_{1}$ of the form $a=t^{j} h$ with $j \neq 0$. This implies that $P \leq K_{1} \leq H$, contradicting our assumption that $\left[K_{1}: P\right]=$ $\infty$. This verifies part (1) of the lemma.

Part (3) follows immediately from part (1). In turn, part (3) immediately implies part (4).

Lemma 5.6 There is an algorithm with the following properties. Suppose we are given a group $G \in \mathcal{M}_{1}$, a subgroup of finite index $G_{1} \leq G$ and a fiber group $H$ of $G_{1}$ so that $G_{1}=H \ltimes\langle t\rangle$. Suppose we are also given a subgroup $P \leq H$ of finite index in $H$ that is normal in $G_{1}$.

The algorithm decides if there is $g \in G-G_{1}$ such that $\left\langle P, g^{-1} P g\right\rangle$ has finite index in $G$ or if for every $g \in G$ the subgroup $P$ has finite index in $\left\langle P, g^{-1} P g\right\rangle$. 
In the former case the algorithm computes all the (finitely many) subgroups $\left\langle P, g^{-1} P g\right\rangle$, where $g \in G$, that have finite index in $G$. It then computes their intersection and finds a normal subgroup $N \leq G$ of finite index in $G$ that is contained in that intersection and such that $N \leq G_{1}$.

In the latter case the algorithm computes the intersection $N_{1}$ of all conjugates of $P$ in $G$. Thus $N_{1}$ is a normal subgroup of $G$ that has finite index in $P$ and in $H$.

Proof We first compute a right transversal $g_{1}=1, g_{2}, \ldots g_{n}$ for $G_{1}$ in $G$. We have already seen in the proof of Lemma [5.5 that each conjugate of $P$ in $G$ has the form $g_{i}^{-1} P g_{i}$. Put $L_{i}:=\left\langle P, g_{i}^{-1} P g_{i}\right\rangle \leq G$. For each $i$ either $P$ has finite index in $L_{i}$ or $L_{i}$ has finite index in $G$. For each $i$ we run the algorithm provided by Lemma 2.2 in parallel with the Todd-Coxeter coset enumeration algorithm for $L_{i}$ to decide which of these alternatives holds.

In particular, we find all of the $L_{i}$ (if any) that have finite index in $G$. If there is at least one such $L_{i}$, we find their intersection $L$, and the intersection $L^{\prime}=L \cap G_{1}$. Then $L^{\prime}$ still has finite index in $G$. We then find the intersection of all conjugates of $L^{\prime}$ in $G$ and denote it by $N$. Clearly $N$ is normal of finite index in $G$, also $N$ is a subgroup of $G_{1}$ and $N$ is contained in all those $L_{i}$ that have finite index in $G$, as required.

Suppose now that all $L_{i}$ turn out to contain $P$ as a subgroup of finite index. Thus each $L_{i}$ is a virtual fiber for $G$. For each of $L_{i}$ we compute its finite presentation. Recall that all conjugates of $P$ in $G$ are of the form $P_{i}:=g_{i}^{-1} P g_{i}$. For each $i$, operating inside $L_{i}$ we compute a generating set for $P_{i}^{\prime}:=P_{i} \cap P$ and rewrite it as the set of words in the generators of $P$. This is possible by Proposition 2.1 since $L_{i}$ is a surface group and thus is locally quasiconvex. Finally, operating inside the surface group $H$, we compute the subgroup

$$
N_{1}:=\cap_{i=1}^{n} P_{i}^{\prime}=\cap_{i=1}^{n} P_{i}^{\prime}=\bigcap_{g \in G} g^{-1} P g .
$$

Then $N_{1}$ is a subgroup of finite index in $H$ and $N_{1}$ is normal in $G$, as required.

Proposition 5.7 There is an algorithm with the following properties. Suppose we are given a group $G \in \mathcal{M}_{1}$, a subgroup of finite index $G_{1} \leq G$, a fiber group $H$ of $G_{1}$ so that $G_{1}=H \ltimes\langle t\rangle$ and a virtual fiber group $H_{1}$ commensurable with $H$.

The algorithm computes the relative rank $\operatorname{rk}_{H_{1}}(G)$ of $G$ with respect to $H_{1}$. 
Proof First we compute a subgroup $P \leq H$ of finite index in $H$ such that $P \leq H_{1}$ and such that $P$ is normal in $G_{1}$. Such a subgroup obviously exists since $H \cap H_{1}$ has finite index in $H$ and hence contains a subgroup $P^{\prime}$ such that $P^{\prime}$ is a characteristic subgroup of finite index in $H$. Since conjugation by $t$ induces an automorphism of $H$, such a subgroup $P^{\prime}$ will be normal in $G_{1}$.

We can algorithmically find some subgroup $P$ with the required properties as follows. First, compute the automorphism $\phi$ of $H$ induced by conjugation by $t$. That is, for each generator $x$ of $H$ express $t^{-1} x t$ as a word in the generators of $H$. Then start enumerating subgroups of finite index $P$ in $H$. For each such $P$ start checking if $P$ is normal in $H$, if $P$ is contained in $H_{1}$ and if $\phi(P)=P$. If yes, then $P$ is as required, and we terminate the process. As we observed above, this algorithm will necessarily terminate since some $P$ with required properties does exist.

We then use the algorithm from Lemma 5.6 and decide if there is $g \in G-G_{1}$ such that $\left\langle P, g^{-1} P g\right\rangle$ has finite index in $G$ or if for every $g \in G$ the subgroup $P$ has finite index in $\left\langle P, g^{-1} P g\right\rangle$.

Suppose first that the former occurs. Then we compute the normal subgroup $N$ of $G$ of finite index in $G$ defined in Lemma 5.6. Denote $\bar{G}=G / N$.

Claim For any set $B \subseteq G$ we have $\left\langle H_{1}, B\right\rangle=G$ if and only if $\left\langle\bar{H}_{1}, \bar{B}\right\rangle=\bar{G}$. Indeed, recall that $N \leq G_{1}$ is normal of finite index in $G$. Since $P$ is normal in $G_{1}$, for every $g \in G$ and every $n \in N$ we have $g^{-1} n^{-1} P n g=g^{-1} P g$ and hence $\left\langle P, g^{-1} P g\right\rangle=\left\langle P, g^{-1} n^{-1} P n g\right\rangle$.

Suppose $\left\langle\bar{H}_{1}, \bar{B}\right\rangle=\bar{G}$. Choose $g \in G$ such that $\left\langle P, g^{-1} P g\right\rangle$ has finite index in $G$. Then there is $n \in N$ such that $g n \in\left\langle H_{1}, B\right\rangle$. Hence $\left\langle P, g^{-1} P g\right\rangle=$ $\left\langle P, g^{-1} n^{-1} P n g\right\rangle$ has finite index in $G$ and therefore contains $N$. But both $P$ and $g n$ are contained in $\left\langle H_{1}, B\right\rangle$. Hence $N \leq\left\langle H_{1}, B\right\rangle$. Since $\left\langle\bar{H}_{1}, \bar{B}\right\rangle=\bar{G}$, this implies that $\left\langle H_{1}, B\right\rangle=G$. This verifies the Claim.

We now compute the image $\bar{H}_{1}$ of $H_{1}$ in the finite group $\bar{G}$ and solve the relative rank problem for $\bar{G}$ with respect to $\bar{H}_{1}$. By the Claim $\operatorname{rk}_{H_{1}}(G)=\operatorname{rk}_{\bar{H}_{1}}(\bar{G})$.

Suppose now that the second alternative of Lemma 5.6 occurs. Then, as in Lemma 5.6 we compute the intersection $N_{1}$ of all conjugates of $P$ in $G$. This is a normal subgroup of $G$ which has finite index in $H$.

Then the group $\widehat{G}:=G / N_{1}$ is virtually cyclic. Since $N_{1} \leq H_{1}$, it is clear that $\operatorname{rk}_{H_{1}}(G)=\operatorname{rk}_{\widehat{H}_{1}}(\widehat{G})$ where $\widehat{H}_{1}$ is the image of $H_{1}$ in $\widehat{G}$. We use then use the algorithm provided by Proposition 5.3 to compute $\operatorname{rk}_{\widehat{H}_{1}}(\widehat{G})$. 


\section{Main technical tool}

In this section we will state the main technical tool needed to prove our main result. This tool was obtained by Kapovich and Weidmann in 22].

If $n \geq 0$ is an integer, for an $n$-tuple $T=\left(g_{1}, \ldots, g_{n}\right)$ of elements of a group $G$ denote $n=l(T)$ and call $n$ the length of $T$.

Let us recall the notion of Nielsen equivalence:

Definition 6.1 Nielsen equivalence Let $T=\left(g_{1}, \ldots, g_{n}\right) \in G^{n}$ be an $n$-tuple of elements of a group $G$. The following moves are called elementary Nielsen moves on $T$ :

(N1) For some $i, 1 \leq i \leq n$ replace $g_{i}$ by $g_{i}^{-1}$ in $T$.

(N2) For some $i \neq j, 1 \leq i, j \leq n$ replace $g_{i}$ by $g_{i} g_{j}$ in $T$.

(N3) For some $i \neq j, 1 \leq i, j \leq n$ interchange $g_{i}$ and $g_{j}$ in $T$.

We say that $T=\left(g_{1}, \ldots, g_{n}\right) \in G^{n}$ and $T^{\prime}=\left(f_{1}, \ldots, f_{n}\right) \in G^{n}$ are Nielsenequivalent in $G$, if there is a chain of elementary Nielsen moves which transforms $T$ to $T^{\prime}$.

Definition 6.2 Partitioned tuple Let $G$ be a group. A partitioned tuple for $G$ is a tuple $M=\left(Y_{1}, \ldots, Y_{s} ; T\right)$ where $s \geq 0$, and where

(a) each of $Y_{i}, T$ is a tuple of elements of $G$;

(b) either $s>0$ or $l(T)>0$;

(c) we have $\left\langle Y_{i}\right\rangle \neq 1$ for each $i>0$.

We call $l\left(Y_{1}\right)+\cdots+l\left(Y_{s}\right)+l(T)$ the length of $M$ and denote it by $l(M)$. We call the $l(M)$-tuple of elements of $G$ obtained by concatenating the tuples $Y_{1}, \ldots, Y_{s}, T$ the underlying tuple of $M$.

Thus $(; T)$ (where $l(T)>0$ ) and $\left(Y_{1} ;\right)$ (where $\left\langle Y_{1}\right\rangle \neq 1$ ) are examples of partitioned tuples.

Definition 6.3 Elementary Moves Let $M=\left(Y_{1}, \ldots, Y_{s} ; T\right)$ be a partitioned tuple for a group $G$. Let $T=\left(t_{1}, \ldots, t_{m}\right)$. The elementary moves on $M$ are the following:

(1) Replace some $Y_{i}$ by $g^{-1} Y_{i} g$ where $g \in\left\langle\left(\cup_{j \neq i} Y_{j}\right) \cup T\right\rangle$. 
(2) Replace some entry $t_{i}$ in $T$ by $u t_{i} u^{\prime}$ where

$$
u, u^{\prime} \in\left\langle\left(\cup_{j=1}^{s} Y_{j}\right) \cup\left\{t_{1}, \ldots, t_{i-1}, t_{i+1}, \ldots, t_{m}\right\}\right\rangle .
$$

Definition 6.4 Equivalence of partitioned tuples Two partitioned tuples $M=\left(Y_{1}, \ldots, Y_{s} ; T\right)$ and $M^{\prime}=\left(Y_{1}^{\prime}, \ldots, Y_{s}^{\prime} ; T^{\prime}\right)$ for a group $G$ are equivalent if there exists a chain of elementary moves taking $M$ to $M^{\prime}$.

It is easy to see that the above definition gives an equivalence relation on the set of partitioned tuples. Moreover, if $M$ and $M^{\prime}$ are equivalent partitioned tuples then $l(M)=l\left(M^{\prime}\right)$ and the underlying tuples of $M, M^{\prime}$ are Nielsen-equivalent in $G$.

Definition 6.5 Complexity of a partitioned tuple We define the complexity of a partitioned tuple $M=\left(Y_{1}, \ldots, Y_{n} ; T\right)$ with $T=\left(t_{1}, \ldots, t_{m}\right)$ to be the pair $(m, n) \in \mathbb{N}^{2}$. We define an order on $\mathbb{N}^{2}$ by setting $(m, n) \leq\left(m^{\prime}, n^{\prime}\right)$ if $m<m$ or if $m=m^{\prime}$ and $n \leq n^{\prime}$. This clearly gives a well-ordering on $\mathbb{N}^{2}$.

Notation 6.6 Invariant sets Let $G$ be a nonelementary torsion-free wordhyperbolic group $G$ with a finite generating set $S$. Let $X$ be the Cayley graph of $G$ with respect to $S$. Let $\delta \geq 1$ be an integer such that $X$ is $\delta$-hyperbolic.

Let $U \leq G$ be a nontrivial subgroup (which is therefore infinite). Let $\Lambda U \subseteq \partial X$ be the limit set of $U$ in $X$.

Let $E(U)$ be the set of all $x \in X$ such that for some $g \in U, g \neq 1$ we have $d(x, g x) \leq 100 \delta$. Let $Z(U)$ be the weak convex hull of $E(U) \cup \Lambda U$, that is $Z(U)$ is the union of all geodesics in $X$ with both endpoints in $E(U) \cup \Lambda U$. Finally, let $X(U)$ denote the closure of $Z(U)$ in $X$.

Note that the definitions of the above $U$-invariant subsets of $X$ depend on the choice of $\delta$.

The following is a corollary of [22, Theorem 2.4]. This is the main technical tool required for our proofs in this paper.

Theorem 6.7 For every integer $k \geq 1$ there exists a computable constant $L=L(k) \geq 0$ with the following property.

Let $G$ be a nonelementary torsion-free word-hyperbolic group with a finite generating set $S$. Let $X$ be the Cayley graph of $G$ with respect to $S$. Let $\delta \geq 1$ be an integer such that $X$ is $\delta$-hyperbolic. 
Let $M=\left(Y_{1}, \ldots, Y_{s} ; T\right)$ by a partitioned tuple for $G$ with $l(M)=k$. Let $U$ be the subgroup generated by the underlying tuple of $M$. Then either

$$
U=\left\langle Y_{1}\right\rangle * \ldots\left\langle Y_{s}\right\rangle * F(T)
$$

or $M$ is equivalent to a partitioned tuple $M^{\prime}=\left(Y_{1}^{\prime}, \ldots, Y_{s}^{\prime} ; T^{\prime}\right)$ such that, denoting $U_{i}^{\prime}=\left\langle Y_{i}^{\prime}\right\rangle$, one of the following occurs:

(1) There are some $i \neq j$ such that $d\left(X\left(U_{i}^{\prime}\right), X\left(U_{j}^{\prime}\right)\right) \leq \delta L(k)$.

(2) There is some $i, 1 \leq i \leq s$ and some element $g$ of $T^{\prime}$ such that

$$
d\left(X\left(U_{i}^{\prime}\right), g X\left(U_{i}^{\prime}\right)\right) \leq \delta L(k) .
$$

(3) There is some element $g$ of $T^{\prime}$ such that $g$ is conjugate in $G$ to an element of length at most $\delta L(k)$.

\section{Generator transfer process}

If $G$ is a group with a finite generating set $S$ and if $g \in G$, we will denote by $|g|_{S}$ the $S$-geodesic length of $g$. Thus $|g|_{S}=d(g, 1)$ in $X(G, S)$. All the constants in this section are assumed to be monotone non-decreasing in each of their integer arguments.

We recall here some results of Kapovich-Weidmann [22].

Lemma 7.1 There exists a computable constant $c_{1}=c_{1}(G, S, \delta, \epsilon)>0$ such that the following holds:

Suppose $G=\langle S \mid R\rangle$ is a finite presentation of a torsion-free word-hyperbolic group $G$ and that $\delta \geq 0$ is an integer such that the Cayley graph $X(G, S)$ is $\delta$ hyperbolic. Let $\epsilon \geq 0$ be an integer and let $U \leq G$ be an infinite subgroup such that $U$ is $\epsilon$-quasiconvex in $X=X(G, S)$. Then $U$ and $X(U)$ are $c_{1}$-Hausdorff close (where $X(U)$ is defined relative $\delta$ ).

Lemma 17.1 follows from [22, Lemma 10.3 and Remark 10.9].

Lemma 7.2 For any integers $K \geq 0$ and $n \geq 1$ there is a computable constant $c_{2}=c_{2}(G, S, \delta, n, K)$ with the following property. Suppose $G=\langle S \mid R\rangle$ is a finite presentation of a group $G$ from the class $\mathcal{M}_{1}$. Suppose $\delta \geq 1$ is an integer such that the Cayley graph $X=X(G, S)$ is $\delta$-hyperbolic. Suppose $U \leq G$ is a non-trivial quasiconvex subgroup $U \leq G$ generated by a set $Y$ with at most $n$ elements such that $Y$ is contained in the $K$-ball around 1 in $\Gamma(G, S)$. Then the sets $U$ and $X(U)$ are $c_{2}$-Hausdorff close (where $X(U)$ is defined relative to $\delta$ ). 
Proof Let $E$ be the maximum of quasiconvexity constants of infinite quasiconvex subgroups generated by subsets with at most $n$-elements from the $K$-ball around 1 in $\Gamma(G, S)$. Put $c_{2}:=c_{1}(G, S, \delta, E)$. Then $c_{2}$ clearly satisfies the requirements of the lemma. In order to see that $c_{2}$ is computable it suffices to see that $E$ is computable. For each $n$-tuple $Y$ of words of length at most $K$ over $S$ apply the algorithm of Proposition 4.6 to decide whether or not $H=\langle Y\rangle$ is quasiconvex in $G$, and if yes, to compute a quasiconvexity constant for $H$. Then $E$ is the maximum of all the quasiconvexity constants obtained in this way and hence $E$ is computable.

Proposition 7.3 There exist a computable constant $c=c\left(G, S, \delta, n_{1}, n_{2}, K\right)$ with the following properties.

Suppose $G=\langle S \mid R\rangle$ is a finite presentation of a group $G$ from the class $\mathcal{M}_{1}$. Suppose $\delta \geq 1$ is an integer such that the Cayley graph $X=X(G, S)$ is $\delta$-hyperbolic. Suppose that $K, n_{1}, n_{2} \geq 1$ are integers.

Suppose $U_{1}=\left\langle Y_{1}\right\rangle$ and $U_{2}=\left\langle g Y_{2} g^{-1}\right\rangle$ are two quasiconvex subgroups of $G$, where $g \in G, Y_{1}=\left(y_{1}, \ldots, y_{n_{1}}\right) \in G^{n_{1}}, Y_{2}=\left(y_{1}^{\prime}, \ldots, y_{n_{2}}^{\prime}\right) \in G^{n_{2}},\left|y_{i}\right|_{S} \leq K$ for $1 \leq i \leq n_{1}$ and $\left|y_{i}^{\prime}\right|_{S} \leq K$ for $1 \leq i \leq n_{2}$. Suppose also that $d\left(X\left(U_{1}\right), X\left(U_{2}\right)\right) \leq$ $K$.

Then the $\left(n_{1}+n_{2}\right)$-tuple $\left(y_{1}, \ldots, y_{n_{1}}, g y_{1}^{\prime} g^{-1}, \ldots, g y_{n_{2}}^{\prime} g^{-1}\right)$ is Nielsen-equivalent to a tuple conjugate in $G$ to $\left(y_{1}, \ldots, y_{n_{1}+n_{2}}\right)$ where $\left|y_{i}\right|_{S} \leq c$ for $1 \leq i \leq$ $n_{1}+n_{2}$.

Proof It is proved in 22 that $c=4 c_{2}(G, S, \delta, n, K)+3 K$ satisfies the requirements of the proposition. The computability of $c_{4}$ now follows from the computability of $c_{2}$ established in Lemma 7.2 .

Proposition 7.4 There is a computable constant $c^{\prime}=c^{\prime}\left(G, S, \delta, n_{1}, n_{2}, K\right)$ with the following properties.

Suppose $G=\langle S \mid R\rangle$ is a finite presentation of a group $G$ from the class $\mathcal{M}_{1}$. Suppose $\delta \geq 1$ is an integer such that the Cayley graph $X=X(G, S)$ is $\delta$-hyperbolic. Suppose that $K, n \geq 1$ are integers.

Let $U=\langle Y\rangle$ where $Y=\left(y_{1}, \ldots, y_{n}\right) \in G^{n}$ and $\left|y_{i}\right| \leq K$ for $1 \leq i \leq n$. Suppose that $U$ is quasiconvex in $G$ and that $d(X(U), g X(U)) \leq K$.

Then the tuple $\left(y_{1}, \ldots, y_{n}, g\right)$ is Nielsen-equivalent to a tuple $\left(y_{1}, \ldots, y_{n}, y_{n+1}\right)$ such that $\left|y_{i}\right|_{S} \leq c^{\prime}$ for $1 \leq i \leq n+1$. 
Proof It is proved in 22] that $c^{\prime}=2 c_{2}(G, S, \delta, n, K)+3 K$ satisfies the requirements of the proposition. By Lemma $7.2 c_{2}$ is computable and therefore $c^{\prime}$ is also computable.

Theorem 7.5 There exists a computable integer constant $C=C(G, S, \delta, k)>$ 0 with the following properties.

Suppose $G=\langle S \mid R\rangle$ be a finite presentation of a group $G$ from the class $\mathcal{M}_{1}$. Suppose $\delta \geq 1$ is an integer such that the Cayley graph $X=X(G, S)$ of $G$ with respect to $S$ is $\delta$-hyperbolic. Suppose $k \geq 1$ is an integer.

Then for any $k$-tuple $T=\left(g_{1}, g_{2}, \ldots, g_{k}\right)$ generating $G$ one of the following holds:

(1) There exists a $k$-tuple $T^{\prime}=\left(g_{1}^{\prime}, g_{2}^{\prime}, \ldots, g_{k}^{\prime}\right)$ Nielsen-equivalent to $T$ such that $\left|g_{i}^{\prime}\right|_{S} \leq C$ for $i=1, \ldots, k$.

(2) There exists a $k$-tuple $T^{\prime}=\left(g_{1}^{\prime}, g_{2}^{\prime}, \ldots, g_{k}^{\prime}\right)$ Nielsen-equivalent to $T$ such that for some $j<k$ the set $\left(g_{1}^{\prime}, \ldots, g_{j}^{\prime}\right)$ generates a virtual fiber of $G$ and $\left|g_{i}^{\prime}\right|_{S} \leq C$ for $i=1, \ldots, j$.

Proof Let $G=\langle S \mid R\rangle$ be a finite presentation of a group from $\mathcal{M}_{1}$ and let $\delta \geq 1$ be an integer such that $X=X(G, S)$ is $\delta$-hyperbolic.

We will prove the result by induction on $k$. For $k=1$ there is nothing to prove, since $G$ is not cyclic. Suppose now that $k \geq 2$ and that the statement has been proved for all $1 \leq k^{\prime}<k$.

We define the constants $R(i)=R(k, i)$ for $i=1, \ldots, k$ inductively as follows. Put $R(1):=\delta L(k)$, where $L(k)$ is the constant provided by Theorem 6.7 For $1<i \leq k$ put

$$
R(i)=\max \left\{R(i-1), c^{\prime}(G, S, \delta, i-1, R(i-1)), \max _{\substack{p+j=i \\ p, j \geq 1}} c(G, S, \delta, p, j, R(i-1))\right\} .
$$

Note that the constants $R(k, i)$ are algorithmically computable.

Let $M=\left(Y_{1}, \ldots, Y_{s} ; H\right)$ be a partitioned tuple of length $k$. Let $n_{i}$ be the length of $Y_{i}$. We will say that $M$ is good if one of the following holds:

(a) each $Y_{j}$ generates a nontrivial quasiconvex subgroup of $G$ and each $Y_{j}$ is conjugate in $G$ to a tuple contained in the ball of radius $R\left(n_{i}\right)$ in $X$.

(b) there is some $Y_{j}$ which generates a virtual fiber of $G$ and such that $Y_{i}$ is conjugate to a tuple contained in the ball of radius $R\left(n_{i}\right)$ in $X$. 
Put $C(G, S, \delta, k):=\max \{C(G, S, \delta, k-1), R(k)\}$. Suppose now that $G$ is generated by a $k$-tuple $T_{0}=\left(g_{1}, \ldots, g_{k}\right)$. If $T_{0}$ is Nielsen-equivalent in $G$ to some tuple containing $1 \in G$, the statement follows from the inductive hypothesis. Therefore we will assume that every $k$-tuple Nielsen-equivalent to $T_{0}$ consists of nontrivial elements of $G$.

Consider a partitioned tuple $M_{0}:=\left(; T_{0}\right)$. Note that $M_{0}$ is good and that the underlying tuple of $M_{0}$ is $T_{0}$. Among all the good partitioned tuples with the underlying tuple Nielsen-equivalent to $T_{0}$ choose a partitioned tuple $M$ of minimal complexity.

Claim We claim that either $M$ has the form $M=\left(Y_{1} ;\right)$ or part (b) of the definition of a good partitioned tuple applies to $M$.

Indeed, suppose not. Then $M=\left(Y_{1}, \ldots, Y_{s} ; T\right)$ where either $s \geq 2$ or $l(T)>0$ and where each $Y_{j}$ generates a quasiconvex subgroup of $G$. Denote $n_{i}=l\left(Y_{i}\right)$ and $n=l(T)$. Recall that the underlying tuple of $M$ is Nielsen-equivalent to $T_{0}$ and hence $n_{1}+\cdots+n_{s}+n=k$.

Since $M$ generates a freely indecomposable group $G$, there exists a partitioned tuple $M^{\prime}=\left(Y_{1}^{\prime}, \ldots Y_{s}^{\prime} ; T^{\prime}\right)$ equivalent to $M$ and such that one of the three cases of Theorem 6.7 applies to $M^{\prime}$. Recall that by definition of equivalence of partitioned tuples each $Y_{i}^{\prime}$ is conjugate to $Y_{i}$ and the underlying tuple of $M^{\prime}$ is Nielsen-equivalent to the underlying tuple of $M$. In particular $M^{\prime}$ is good, since $M$ was good. Put $U_{i}=\left\langle Y_{i}^{\prime}\right\rangle$.

Suppose that Case (1) of Theorem 6.7 applies to $M^{\prime}$. Without loss of generality we may assume that $d\left(X\left(U_{1}\right), X\left(U_{2}\right)\right) \leq L(k) \delta$. Recall that since $M$ and $M^{\prime}$ are good, each $Y_{i}^{\prime}$ is conjugate to a tuple contained in the ball of radius $R\left(n_{i}\right)$ in $X$. Then by Proposition 7.3 the tuple $\left(Y_{1}^{\prime}, Y_{2}^{\prime}\right)$ is Nielsen-equivalent to an $\left(n_{1}+n_{2}\right)$-tuple $Y_{0}^{\prime \prime}$ such that $Y_{1}^{\prime \prime}$ is conjugate to a set contained in the ball of radius $c=c\left(G, S, \delta, n_{1}, n_{2}, \max \left\{L(k) \delta, R\left(n_{1}\right), R\left(n_{2}\right)\right\}\right)$ in $X$. Recall that by definition of $R(i)$ we have $R(1)=L(k) \delta \leq R\left(n_{1}\right), R\left(n_{2}\right) \leq R\left(n_{1}+n_{2}-1\right)$. Therefore $Y_{1}^{\prime \prime}$ is conjugate to a set contained in the ball of radius $R\left(n_{1}+n_{2}\right)$. Consider now a partitioned tuple $M^{\prime \prime}:=\left(Y_{1}^{\prime \prime}, Y_{3}^{\prime}, \ldots, Y_{s}^{\prime} ; T^{\prime}\right)$. Then $M^{\prime \prime}$ is good and the underlying tuple of $M^{\prime \prime}$ is Nielsen-equivalent to $T_{0}$. On the other hand the complexity of $M^{\prime \prime}$ is smaller than the complexity of $M$, which contradicts the minimal choice of $M$.

Suppose now that Case (2) of Theorem 6.7 applies to $M^{\prime}$. Without loss of generality we may assume that for some entry $g$ of $T^{\prime}$ we have $d\left(X\left(U_{1}\right), g X\left(U_{1}\right)\right) \leq$ $L(k) \delta$. Then by Proposition 7.4 the $\left(n_{1}+1\right)$-tuple $\left(Y_{1}^{\prime}, h\right)$ is Nielsen-equivalent to an $\left(n_{1}+1\right)$-tuple $Y_{1}^{\prime \prime}$ which is conjugate to a subset contained in the ball 
of radius $c^{\prime}=c^{\prime}\left(G, S, \delta, n_{1}, \max \left\{R\left(n_{1}\right), L(k) \delta\right\}\right) \leq R\left(n_{1}+1\right)$ in $X$. Put $M^{\prime \prime}:=\left(Y_{1}^{\prime \prime}, Y_{2}^{\prime}, \ldots, Y_{s}^{\prime} ; T^{\prime \prime}\right)$ where $T^{\prime \prime}$ is obtained from $T^{\prime}$ by deleting the entry $g$. Then $M^{\prime \prime}$ is good and the underlying tuple of $M^{\prime \prime}$ is Nielsen-equivalent to $T_{0}$. On the other hand the complexity of $M^{\prime \prime}$ is smaller than that of $M$, which contradicts the minimal choice of $M$.

Suppose that Case (3) of Theorem 6.7 applies to $M^{\prime}$. Then $T^{\prime}$ contains an element $g$ that is conjugate in $G$ to an element of length at most $L(k) \delta$. Put $M^{\prime \prime}:=\left(Y_{1}^{\prime}, \ldots Y_{s}^{\prime}, Y_{s+1} ; T^{\prime \prime}\right)$ where $Y_{s+1}=(g)$ and where $T^{\prime \prime}$ is obtained from $T^{\prime}$ by deleting the entry $g$. Then $M^{\prime \prime}$ is good and the underlying tuples of $M^{\prime}$ and $M^{\prime \prime}$ coincide. In particular, the underlying tuple of $M^{\prime \prime}$ is Nielsenequivalent to $T_{0}$. On the other hand the complexity of $M^{\prime \prime}$ is smaller than that of $M$, which contradicts the minimal choice of $M$.

Thus we have verified the Claim. This immediately implies the statement of the theorem.

\section{The rank problem}

In this section we give a proof of Theorem $\mathrm{A}$

Theorem 8.1 There is an algorithm that, given a finite presentation $G=$ $\langle S \mid R\rangle$ of a group $G$ from the class $\mathcal{M}_{1}$, computes the rank of $G$.

Proof Let $G=\langle S \mid R\rangle$ be a finite presentation of $G \in \mathcal{M}_{1}$. Denote $X=$ $X(G, S)$, the Cayley graph of $G$ with respect to $S$. First we compute an integer $\delta \geq 1$ such that $X$ is $\delta$-hyperbolic. Let $k=\# S$. We next compute the constant $C=C(G, S, \delta, k)>0$ provided by Theorem 7.5. We then enumerate all $p$-tuples $T$ of words over $S$ with $1 \leq p \leq k$, where every word has length at most $C$. Let $\mathcal{T}$ be the set of all such tuples.

For each tuple $T \in \mathcal{T}$ we use Proposition 4.6 to decide if $H_{T}:=\langle T\rangle$ is quasiconvex in $G$ or if $H_{T}$ is a virtual fiber of $G$. If $H_{T}$ is a virtual fiber of $G$ we compute the rank of $H_{T}$ and, using Proposition [5.7, the relative $\operatorname{rank} \operatorname{rk}_{H_{T}}(G)$ of $G$ with respect to $H_{T}$. If $H_{T}$ is quasiconvex in $G$, we check whether or not $H_{T}=G$, that is $\langle T\rangle=G$.

Let $p \leq k$. Theorem 7.5 implies that $\operatorname{rk}(G) \leq p$ if and only if either there exists a $p$-tuple $T \in \mathcal{T}$ such that $G=\langle T\rangle$ or there exists a tuple $T \in \mathcal{T}$ generating a virtual fiber $H_{T}$ such that $\operatorname{rk}\left(H_{T}\right)+\operatorname{rk}_{H_{T}}(G) \leq p$.

Therefore for each $0 \leq p \leq k$ we can decide whether or not $\operatorname{rk}(G) \leq p$. The smallest $0 \leq p \leq k$ such that $\operatorname{rk}(G) \leq p$ is the $\operatorname{rank}$ of $G$. 
Theorem 8.2 There is an algorithm that, given a finite presentation $G=$ $\langle S \mid R\rangle$ of a group $G$ from the class $\mathcal{M}$, computes the rank of $G$.

Proof We first apply the algorithm from Proposition 3.3 and compute a decomposition

$$
G=G_{1} * \cdots * G_{s} * F_{r}
$$

where each $G_{i}$ is one-ended and is given to us by an explicit finite presentation.

By Proposition 3.2 each $G_{i} \in \mathcal{M}_{1}$. We use the algorithm from Theorem 8.1 and compute $\operatorname{rk}\left(G_{i}\right)$ for each $i$.

Then by Grushko's Theorem it follows that $\operatorname{rk}(G)=\operatorname{rk}\left(G_{1}\right)+\cdots+\operatorname{rk}\left(G_{s}\right)+r$.

\section{$9 \quad$ Nielsen equivalence classes of generating tuples}

In this section we prove Theorem B from the Introduction.

Theorem 9.1 Let $G$ be a group from the class $\mathcal{M}$. Then for each $k \geq 1$ there exists only finitely many Nielsen-equivalence classes of $k$-tuples generating $G$.

Proof First, observe that it suffices to prove Theorem 9.1 for groups from the class $\mathcal{M}_{1}$. Indeed, Grushko's theorem (see the proofs, for example, in [19, 33]) ensures that every tuple generating a group $G_{1} * G_{2}$ is Nielsen equivalent to a tuple $\left(T_{1}, T_{2}\right)$ where $T_{1}$ generates $G_{1}$ and $T_{2}$ generates $G_{2}$. Note also that the statement of Theorem 9.1 holds for infinite cyclic groups (and, moreover, for finitely generated free groups).

Thus assume that $G=\langle S \mid R\rangle \in \mathcal{M}_{1}$. Theorem 7.5 implies that there is a constant $C>0$ such that if $T=\left(g_{1}, \ldots, g_{k}\right)$ generates $G$ than either there exists a $k$-tuple $T^{\prime}=\left(y_{1}, y_{2}, \ldots, y_{k}\right)$ Nielsen-equivalent to $T$ such that $\left|y_{i}\right|_{S} \leq$ $C$ for $i=1, \ldots, k$, or there exists a $k$-tuple $T^{\prime}=\left(y_{1}, y_{2}, \ldots, y_{k}\right)$ Nielsenequivalent to $T$ such that for some $j<k$ the set $\left(y_{1}, \ldots, y_{j}\right)$ generates a virtual fiber $U$ of $G$ and $\left|y_{i}\right|_{S} \leq C$ for $i=1, \ldots, j$.

In the former case we are done, so suppose that the latter occurs. Thus there is a subgroup $G_{1}$ of finite index in $G$ such that $G_{1}=H \rtimes\langle t\rangle$ and that the fiber $H$ of $G_{1}$ is commensurable with $U$. By Remark 4.4 we may assume that $G_{1}$ is normal in $G$, since a finite cover of a closed 3-manifold fibering over a circle also fibers over a circle and the two fiber groups are commensurable.

Put $H_{U}:=H \cap U$. Note that $H_{U}$ is commensurable with both $H$ and $U$. 
There is a subgroup $P \leq H_{U}$ of finite index such that $P$ is normal in $G_{1}$. Then by Lemma 5.5 there are only finitely many conjugates of $H$ and of $P$ in $G$. Hence there are only finitely many conjugates of $H_{U}$ in $G$.

Note also that since there are only finitely many subgroups $U$ of $G$ under consideration, only finitely many possibilities for $G_{1}, H$ and $H_{U}$ arise.

We consider the following two cases:

Case 1 The subgroup $H_{U}$ is commensurable with $g^{-1} H_{U} g$ for every $g \in G$. Then the group $N=\cap_{g \in G} g^{-1} H_{U} g$ is a subgroup of finite index in $H_{U}$ and in $U$ that is normal in $G$. Therefore the group $G / N$ is virtually cyclic.

The same argument as in the proof of the solvability of the relative rank problem with respect to virtual fibers (see Proposition 5.3 and Remark [5.4) now yields the conclusion that there are only finitely many Nielsen-equivalence classes of $k$-tuples $T^{\prime}=\left(y_{1}, y_{2}, \ldots, y_{k}\right)$ with these properties.

Case 2 Suppose that there is some $g \in G$ such that $H_{U}$ is not commensurable with $g H_{U} g^{-1}$. Recall that $U$ is generated by $\left(y_{1}, \ldots, y_{j}\right)$ and that $U$ is commensurable with $H_{U}$. Thus there is $q>j$ such that $H_{U}$ is not commensurable with $y_{q}^{-1} H_{U} y_{q}$. Note that $y_{q}^{-1} H_{U} y_{q} \leq G_{1}$ since $G_{1}$ is normal in $G$. Recall also that $H_{U}$ has only finitely many conjugates in $G$ and hence only finitely many possibilities for $y_{q}^{-1} H_{U} y_{q}$ arise.

The subgroup $H_{U}$ contains a subgroup $P$ of finite index in both $H$ and $H_{U}$ such that $P$ is normal in $G_{1}$. Hence by Lemma 5.5 every subgroup containing $P$ is either commensurable with $H_{U}$ or has finite index in $G$. By assumption $y_{q}^{-1} H_{U} y_{q}$ is not commensurable with $H_{U}$. Hence $L:=\left\langle H_{U}, y_{q}^{-1} H_{U} y_{q}\right\rangle$ has finite index in $G_{1}$ and in $G$. Indeed, otherwise $L$ is a finite extension of $H_{U}$ and $L$ contains a virtual fiber $y_{q}^{-1} H_{U} y_{q}$. Since virtual fibers are surface groups, whenever one of them is contained in another, it must have finite index in this bigger virtual fiber. Hence $y_{q}^{-1} H_{U} y_{q}$ is a subgroup of finite index in $L$. Since $H_{U}$ also has finite index in $L$, this implies that $H_{U}$ is commensurable with $y_{q}^{-1} H_{U} y_{q}$, contrary to our assumptions. Thus indeed $L$ has finite index in $G_{1}$.

Write $y_{q}$ as $y_{q}=g^{\prime} h t^{s}$ where $g^{\prime}$ is an element from a fixed finite transversal for $G_{1}$ in $G$, where $h \in H$ and $s \in \mathbb{Z}$. Since $L \leq G_{1}$ has finite index in $G_{1}$, we conclude that $y_{q}^{-1} H_{U} y_{q}$ is not contained in $H$. Therefore there is $h_{u} \in H_{U}$ such that $y_{q}^{-1} h_{u} y_{q}=h^{\prime} t^{a}$ where $h^{\prime} \in H$ and $a \neq 0$. Then

$$
\left(g^{\prime} h\right)^{-1} h_{u}\left(g^{\prime} h\right)=t^{s} h^{\prime} t^{a} t^{-s}=h^{\prime \prime} t^{a} \quad \text { where } h^{\prime \prime} \in H .
$$

Hence for every integer $m$

$$
\left(g^{\prime} h\right)^{-1} h_{u}^{m}\left(g^{\prime} h\right)=h_{m} t^{a m} \quad \text { where } h_{m} \in H,
$$


that is

$$
h_{u}^{m} g^{\prime} h=g^{\prime} h h_{m} t^{a m} .
$$

Put

$$
y_{q}^{\prime}=h_{u}^{m} y_{q}=h_{u}^{m} g^{\prime} h t^{s}=g^{\prime} h h_{m} t^{a m+s}=g^{\prime} t^{a m+s} h_{m}^{\prime}
$$

where $h_{m}^{\prime} \in H$. We can choose an appropriate $m$ to ensure that $|a m+s| \leq|a|$.

Replacing $y_{q}$ by $y_{q}^{\prime}=h_{u} y_{q}$ in $T^{\prime}$ results in a Nielsen-equivalent tuple since $h_{u} \in H_{U} \leq U$. After this we can use the subgroup of finite index $L$ in $G$ to make all the entries $y_{i}$ for $i>j, i \neq q$ short. Next, using the fact that $H_{U}$ has finite index in $H$, we multiply $h_{q}^{\prime}=g^{\prime} t^{a m+s} h_{m}^{\prime}$ on the right by an appropriate element of $H_{U}$ to make this entry short as well.

\section{References}

[1] I Agol, Tameness of hyperbolic 3-manifolds, arXiv:math.GT/0405568

[2] J Alonso, T Brady, D Cooper, V Ferlini, M Lustig, M Mihalik, M Shapiro, H Short, Notes on hyperbolic groups, from: "Group theory from a geometric viewpoint (Trieste 1990)", World Scientific, River Edge, NJ (1991) 3-63 MathReview

[3] G Arzhantseva, On quasiconvex subgroups of word hyperbolic groups Geom. Dedicata 87 (2001) 191-208 MathReview

[4] G Arzhantseva, A Yu Ol'shanskii, Generality of the class of groups in which subgroups with a lesser number of generators are free Mat. Zametki 59 (1996) 489-496, 638 (Russian); translation in Math. Notes 59 (1996) 350-355 MathReview

[5] G Baumslag, C F Miller III, H Short, Unsolvable problems about small cancellation and word hyperbolic groups Bull. London Math. Soc. 26 (1994) 97101 MathReview

[6] M Boileau, H Zieschang, Heegaard genus of closed orientable Seifert 3manifolds Invent. Math. 76 (1984) 455-468 MathReview

[7] D Calegari, D Gabai, Shrinkwrapping and the taming of hyperbolic 3manifolds, arXiv:math.GT/0407161

[8] R Canary, A covering theorem for hyperbolic 3-manifolds and its applications, Topology 35 (1996) 751-778 MathReview

[9] N Dunfield, W P Thurston, The virtual Haken conjecture: Experiments and examples, Geom. Topol. 7 (2003) 399-441 MathReview

[10] D B A Epstein, J W Cannon, D F Holt, S V F Levy, M S Paterson, W P Thurston, Word processing in groups, Jones and Bartlett Publishers, Boston, MA (1992) MathReview 
Kleinian groups and the rank problem

[11] D Gabai, Convergence groups are Fuchsian groups, Ann. of Math. 136 (1992) 447-510 MathReview

[12] É Ghys, P de la Harpe (editors), Sur les groupes hyperboliques d'après Mikhael Gromov, Progress in Mathematics 83, Birkhäuser Boston Inc. Boston, MA, USA (1990) MathReview

[13] V Gerasimov, Detecting connectedness of the boundary of a hyperbolic group, preprint (1999)

[14] S M Gersten, H Short, Rational subgroups of biautomatic groups, Ann. of Math. 134 (1991) 125-158 MathReview

[15] M Gromov, Hyperbolic groups, from: "Essays in group theory" (SM Gersten, editor), Math. Sci. Res. Inst. Publ. 8, Springer-Verlag (1987) 75-263 MathReview

[16] W Jaco, H Rubinstein, 0-efficient triangulations of 3-manifolds, J. Differential Geom. 65 (2003) 61-168 MathReview

[17] K Johannson, Topology and combinatorics of 3-manifolds, Lecture Notes in Mathematics 1599, Springer-Verlag, Berlin, Germany (1995) MathReview

[18] I Kapovich, Detecting quasiconvexity: algorithmic aspects, from: "Geometric and computational perspectives on infinite groups (Minneapolis, MN and New Brunswick, NJ, 1994)", DIMACS Ser. Discrete Math. Theoret. Comput. Sci. 25, Amer. Math. Soc., Providence, RI, USA (1996) 91-99 MathReview

[19] I Kapovich, A Myasnikov, R Weidmann, Foldings, graphs of groups and the membership problem, Internat. J. Alg. Comput. 15 (2005) 95-128

[20] I Kapovich, H Short, Greenberg's theorem for quasiconvex subgroups of word hyperbolic groups, Canad. J. Math. 48 (1996) 1224-1244 MathReview

[21] I Kapovich, R Weidmann, Nielsen Methods for groups acting on hyperbolic spaces, Geom. Dedicata 98 (2003) 95-121 MathReview

[22] I Kapovich, R Weidmann, Freely indecomposable groups acting on hyperbolic spaces, Internat. J. Algebra Comput. 14 (2004) 115-171 MathReview

[23] M Kapovich, Hyperbolic manifolds and discrete groups, Progress in Mathematics 183, Birkhäuser, Boston, MA, USA (2001) MathReview

[24] A Karrass, W Magnus, D Solitar, Combinatorial Group Theory: Presentations of groups in terms of generators and relations, Interscience Publishers [John Wiley and Sons, Inc.], New York-London-Sydney (1966) MathReview

[25] P Papasoglu, An algorithm detecting hyperbolicity, from: "Geometric and computational perspectives on infinite groups (Minneapolis, MN and New Brunswick, NJ 1994)", DIMACS Ser. Discrete Math. Theor. Comput. Sci. 25, Amer. Math. Soc. Providence, RI, USA (1996) 193-200 MathReview

[26] E Rips, Subgroups of small cancellation groups, Bull. Lond. Math. Soc. 14 (1982) 45-47 MathReview 
[27] J Rubinstein, Polyhedral minimal surfaces, Heegaard splittings and decision problems for 3-dimensional manifolds, from: "Geometric topology (Athens, GA, 1993)", AMS/IP Stud. Adv. Math. 2, Amer. Math. Soc. Providence, RI, USA (1997) 1-20 MathReview

[28] P Schupp, Coxeter groups, 2-completion, perimeter reduction and subgroup separability, Geom. Dedicata 96 (2003) 179-198 MathReview

[29] Z Sela, The isomorphism problem for hyperbolic groups I, Ann. of Math. 141 (1995) 217-283 MathReview

[30] C Sims, Computation with finitely presented groups, volume 48 of Encyclopedia of Mathematics and its Applications, Cambridge University Press, Cambridge (1994) MathReview

[31] J Souto, The rank of the fundamental group of hyperbolic 3-manifolds fibering over the circle, preprint (2005)

[32] P Tukia, Homeomorphic conjugates of Fuchsian groups, J. Reine Angew. Math. 391 (1988) 1-54 MathReview

[33] R Weidmann, The Nielsen method for groups acting on trees, Proc. London Math. Soc. 85 (2002) 93-118 MathReview

[34] R Weidmann, The rank problem for sufficiently large Fuchsian groups, preprint (2004) 